\title{
Physical and chemical properties of meteoroids as deduced from observations
}

\author{
Jiří Borovička
}

Astronomical Institute of the Academy of Sciences, 25165 Ondřejov, Czech Republic

email: borovic@asu.cas.cz

\begin{abstract}
A review of the current knowledge of physical properties and chemical composition of meteoroids entering the Earth's atmosphere is presented. Meteoroid penetration ability, ablation coefficients, beginning heights, light curves, fragmentation, and spectra are considered. The inferred bulk densities, mechanical strengths, rotation, and atomic elemental abundances are discussed. Cometary meteoroids are effectively grain aggregates with low bulk density (100-1000 kg $\mathrm{m}^{-3}$ ), high porosity and low cohesivity. A volatile matrix holding the grains together may be present. Presence of large amounts of organic material is not firmly established. Small chunks $(\sim 1 \mathrm{~mm})$ of denser material are sometimes contained in cometary meteoroids. Chemically, cometary grains are similar to CI chondrites but there is a hint of enhancement of $\mathrm{Na}, \mathrm{Si}$, and $\mathrm{Mg}$ and depletion of $\mathrm{Fe}, \mathrm{Cr}$, and $\mathrm{Mg}$. Larger chemical diversity is observed among small meteoroids on cometary orbits not belonging to meteoroid streams. The relatively frequent Na-free meteoroids are probably fragments of cometary irradiation crust. Asteroidal meteoroids exhibit much lower mechanical strengths than stony meteorites, clearly due to the presence of large scale cracks. Iron meteoroids dominate among asteroidal meteoroids smaller than $1 \mathrm{~cm}$.
\end{abstract}

Keywords. meteors, meteoroids

\section{Introduction}

All solid bodies in the interplanetary space larger than dust particles and smaller than asteroids are called meteoroids. Meteoroids occupy a significant part of the size spectrum of solar system bodies, from tens of microns to about ten meters. The orbital lifetime of meteoroids in the inner solar system is much shorter than the age of the solar system. Meteoroid population must be therefore continuously replenished. The main sources of meteoroids are asteroids and comets. Only a tiny part comes from the solid surfaces of planets and satellites and from interstellar space. The main motivation of meteoroid study is recognition of different meteoroid populations, establishing of their properties, and contributing this way to the study of asteroids and comets. Another aspect is the direct influence of meteoroids on terrestrial environment.

Meteoroids are too small to be observed remotely in interplanetary space. Only large concentrations of meteoroids can be revealed remotely but these observations provide limited information on meteoroid properties. Very small meteoroids are part of the solar system dust structures such as zodiacal dust cloud and asteroidal dust bands. Larger meteoroids of millimeter to centimeter range form dust trails in the orbits of many short period comets. An encounter of the Earth with a dust trail will manifest itself as a meteor storm.

The terrestrial atmosphere is, in fact, our best detector of meteoroids. The process of meteoroid disintegration in the atmosphere generates electromagnetic radiation including visible light and leads to the formation of an ionized trail. Large meteoroids produce also sonic waves. Since up to $7 \times 10^{5} \mathrm{~km}^{2}$ of atmosphere can be sampled from a single site (for meteor height of $100 \mathrm{~km}$ and viewing more than 10 degrees above horizon), small and 
medium sized meteoroids can be effectively studied from one ground station (although stereoscopic observations from two stations are needed for the determination of meteor trajectory). The study of large meteoroids which enter the atmosphere only rarely must rely on long-term observations using whole networks of stations, or, in the future, on space-borne monitoring of the atmosphere on global scale.

Under favorable circumstances, small part of a meteoroid can survive the atmospheric entry and land as a meteorite. Laboratory studies of meteorites provide, of course, the most detailed information on meteoroid properties. They cover, however, only small part of the whole meteoroid population, since only meteoroids large enough, strong enough, and entering the atmosphere with sufficiently low velocity can drop meteorites.

The atmosphere of the Earth is not the only possible meteoroid detector. Meteoroid impacts have been also detected on the surface of Moon (Cudnik et al. 2003) and meteors have been observed in the atmospheres of Mars (Selsis et al. 2005) and Jupiter (Cook \& Duxbury 1981). A meteorite has been recently found on the surface of Mars (Arvidson \& Squyres 2005). Nevertheless, in this paper we will deal only with the meteoroids in the terrestrial atmosphere. Mars, in particular, has a good potential of studying meteoroids closer to the asteroid belt but at the moment the data are very scarce.

Though the terrestrial atmosphere is an efficient detector of meteoroids, inferring physical and chemical properties of incoming meteoroids from remote meteor observation is not a trivial task. In this paper I will review the current state of the problem and outline the open questions. I will concentrate on information gained from the optical observation which provide most complex data. Meteor heights, light curves, deceleration and spectra provide the basis for meteoroid studies. At the same time, pre-encounter heliocentric orbits can be derived. In the case that the meteoroid belongs to a meteor shower, it can be directly linked to the parent body of the shower (if known). This way, various objects can be studied: comet $2 \mathrm{P} /$ Encke, Jupiter family comets (e.g. 21P/Giacobini-Zinner), Halley type comets (e.g. 109P/Swift-Tuttle and 55P/Tempel-Tuttle), long period comets (e.g. 1861 I Thatcher), as well as some asteroids (3200 Phaethon and 2003 EH1). For the majority of asteroids, however, no meteoroid streams have been revealed.

\section{Classification and ablation of large meteoroids}

In the following, I will divide the discussion in two parts, taking separately the "large" and "small" meteoroids. There are at least two reasons for this. First, large meteoroids, larger than about one centimeter in diameter, produce bright meteors (fireballs) which are observed by different techniques than normal meteors. More importantly, large meteoroids spend longer time in the atmosphere before being disintegrated and give rise to more phenomena. In contrast to small meteoroids, they can be significantly decelerated, produce more complex light curves, and can drop meteorites in some cases. From the physical point of view, large meteoroids are loosing most of their mass in the continuum flow regime while small meteoroids are subject to free molecule flow regime of transition flow regime (Popova 2005). There are also evidences that asteroidal and cometary meteoroids are represented in different proportions among small and large meteoroids.

\subsection{Classification}

Meteoroids differ very much in their ability to penetrate the atmosphere. An example is given in Fig. 1, where the light curves two very bright fireballs are plotted. The Šumava and Benešov fireballs are among the brightest well observed fireballs. Their initial velocity was not very different $\left(27\right.$ and $21 \mathrm{~km} \mathrm{~s}^{-1}$ ) and the initial meteoroid mass was of the order of several metric tons in both cases. The behavior in the atmosphere was very different, 


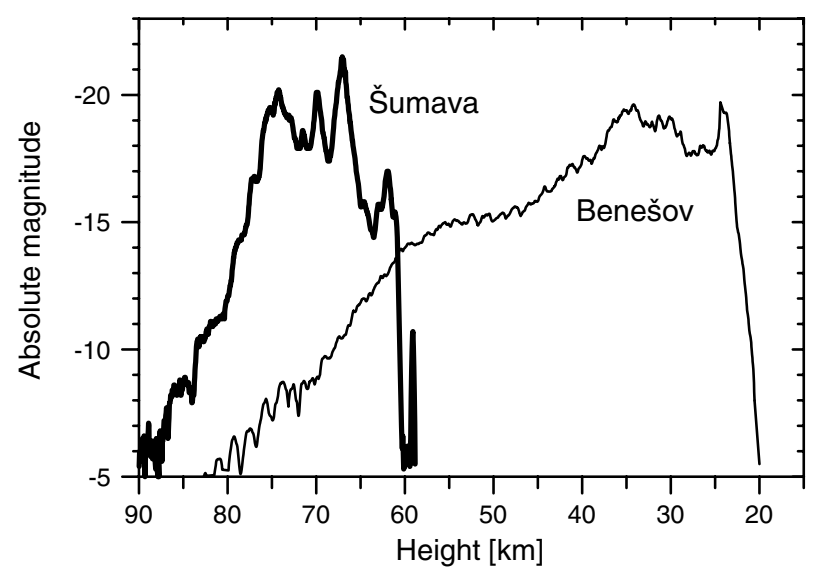

Figure 1. The dependence of brightness on atmospheric height for two very bright fireballs. According to Borovička \& Spurný (1996).

nevertheless. Šumava reached the maximum brightness $(-21.5 \mathrm{mag})$ at the height of $67 \mathrm{~km}$, where Benešov was still in process of initial increase of luminosity. The Sumava body was completely destroyed at $58 \mathrm{~km}$, while Benešov radiated below $20 \mathrm{~km}$ of height. The difference in terms of air density is by a factor of 400 .

The conspicuous differences in fireball end heights form the basis of the classical fireball classification formed by Ceplecha and McCrosky (1976). An empirical PE criterion was created which relates the end height with initial mass, initial velocity and the slope of the trajectory. The fireballs were divided into four groups (or types) which differ greatly in the ablation ability. The most resistant group I was identified with ordinary chondritic or, more generally, stony meteoritic material. This relation is confirmed by the fact that photographed meteorite falls Lost City, Innisfree and Neuschwanstein belong to group I. The first two are ordinary chondrites, the last one is an enstatite chondrite. Group II corresponds to more fragile carbonaceous material. The group IIIA and the most fragile group IIIB are both of cometary origin as evidenced by the fact typical cometary shower fireballs belong to these groups. Perseid fireballs are of type IIIA (Spurný 1995), while most Leonids observed in 1998 were classified as IIIB, although many of them were close to the boundary with IIIA (Spurný et al. 2000a). The typical IIIB material is represented by the October Draconids (Ceplecha \& McCrosky 1976), which were found to be unusually soft already by Jacchia et al. (1950).

The PE criterion is simple and easy to use since only basic geometric and photometric reduction of the data is necessary (note that the original luminous efficiency of Ceplecha \& McCrosky 1976 must be used for computing the photometric mass). The classification based on the PE criterion is still valid today. The obvious question, however, is what physical and/or chemical properties of meteoroids cause their different behavior in the atmosphere. To proceed further with this question, we must refer to the so called physical theory of meteors.

\subsection{Ablation and deceleration}

The meteoroid dynamics in the atmosphere is governed by two differential equations - the drag equation and the mass-loss equation (see e.g. Ceplecha et al. 1998). The equations contain two independent parameters, the ablation coefficient, $\sigma$, defined as $\sigma=\Lambda /(2 \xi \Gamma)$, and the shape-density coefficient $K$ defined as $K=\Gamma A \delta^{-2 / 3}$. Here $\Lambda$ is the heat transfer coefficient, $\xi$ is the energy necessary for ablation of a unit mass, $\Gamma$ is the drag coefficient, 
Table 1. Average apparent ablation coefficients for fireball groups (Ceplecha et al. 1993).

\begin{tabular}{lcc}
\hline Group & Ablation coefficient $\left[\mathrm{s}^{2} \mathrm{~km}^{-2}\right]$ & No. of cases \\
\hline I & 0.02 & 33 \\
II & 0.05 & 14 \\
IIIA & 0.14 & 3 \\
IIIB & 0.6 & 1 \\
\hline
\end{tabular}

$A$ is the shape coefficient (relating cross-section to volume), and $\delta$ is the bulk density of the meteoroid. Assuming that $\sigma$ and $K$ are constant and the meteoroid does not suffer fragmentation, and knowing the slope of the trajectory, the observed dynamics of the meteoroid can be fitted. The fit provides the initial velocity, $v_{\infty}$, and the value of $\sigma$. The initial mass, $m_{\infty}$, and $K$ cannot be separated and only the product $K m_{\infty}^{-1 / 3}$ is obtained.

The ablation coefficient characterizes the ability of the meteoroid to ablate and can be used to quantify the meteoroid classification. There is strong relation between $\sigma$ and the meteoroid type according to the PE criterion. Usually, $\sigma$ is given in $\mathrm{s}^{2} \mathrm{~km}^{-2}$, which is equivalent to $\mathrm{kg} \mathrm{MJ}^{-1}$. The values of the ablation coefficient computed without considering meteoroid fragmentation are called apparent (Ceplecha \& ReVelle 2005). In many cases, in particular for well observed type I fireballs, the assumption of no-fragmentation is unsatisfactory since the fireball dynamics cannot be fitted well. This allowed Ceplecha et al. (1993) to modify the method for the case of gross-fragmentation, when a sudden mass loss occurs at one point. Taking the gross-fragmentation into account, the resulting ablation coefficients (which describe the mass loss outside fragmentation) decreased. Nevertheless, in Table 1 we present the average apparent ablation coefficients for different fireball groups taken from non-fragmentation solutions of Ceplecha et al. (1993). These values show the differences between the groups most clearly. Unfortunately, the dynamic method could be used only for few fireballs of groups III, because these bodies suffer only little deceleration.

\subsection{Light curves and the intrinsic ablation coefficient}

To learn more about the meteoroid properties and the process of fragmentation, we can use the information from fireball light curves. According to the physical theory of meteors, the instantaneous meteor luminosity is proportional to the loss of meteoroid kinetic energy (the proportionality factor is called luminous efficiency, $\tau$ ). In most cases, the energy loss is dominated by mass loss, not deceleration. Fireballs of types IIIA and IIIB often exhibit brief increases of brightness called flares. The flares are caused by the loss of large amount of material in the form of tiny dust particles which evaporate quickly and their energy is radiated out. This was demonstrated directly on an instantaneous photograph showing the correlation of fireballs flare, splitting into two pieces, and formation of long wake made of tiny fragments (Konovalova 2003). Sometimes, the fireball trajectory terminates by a flare caused by complete pulverization of the meteoroid. Fireballs of types I and II do not show bright flares so often. They fragment preferably into macroscopic fragments and smaller amount of dust is released.

Borovička \& Spurný (1996) analyzed the light curve of the very bright IIIB fireball Sumava (see Fig. 1). The body of estimated initial mass of $5000 \mathrm{~kg}$ and diameter of $4.5 \mathrm{~m}$ exhibited five flares, the brightest one having an amplitude of 4 magnitudes, corresponding to 40 fold increase of brightness in 0.1 second. The apparent ablation coefficient of the meteoroid was $0.32 \mathrm{~s}^{2} \mathrm{~km}^{-2}$. Nevertheless, the light curve analysis showed that more than $85 \%$ of initial mass was lost in five major breakups. During each breakup, only $5 \%$ 
of lost mass was released in form of macroscopic fragments, the rest was lost in form of dust.

Recently Ceplecha \& ReVelle (2005) presented an elaborated fragmentation model (FM) which is able to explain simultaneously fireball dynamics and light curve to amazing details. The parameters $\sigma, K$, and $\tau$ are taken as variable and two types of fragmentation are considered - into several large fragments and into cluster of small fragments. The controversial aspects of the model formalism is that it allows for "flares" of duration of several seconds and for enormous variations of $K$, which are difficult to interpret physically. On the other hand, the model for the first time distinguishes the apparent and intrinsic values of the ablation coefficient and luminous efficiency. The intrinsic values are corrected for the effect of fragmentation and express therefore the meteoroid ablation properties other than fragmentation ability. The surprising result is that the intrinsic ablation coefficient lies the range $0.001-0.008 \mathrm{~s}^{2} \mathrm{~km}^{-2}$ in most fireballs including types IIIA and IIIB and the variations from fireball to fireball do not exceed the variations inside one fireball. Only rarely the intrinsic $\sigma$ reaches $0.015 \mathrm{~s}^{2} \mathrm{~km}^{-2}$, which is still less than the typical apparent values. Fragmentation is therefore the dominant process of mass loss for all types of meteoroids. It is more efficient than the evaporation or melting of the meteoroid surface. Moreover, there are no differences in the evaporation properties of meteoroids of various types. The huge differences in the ablation abilities are caused by mechanical properties of the material rather than its composition. This suggests that the cometary meteoroids differ from ordinary chondrites mostly by their bulk density, porosity and mechanical strength.

It is interesting to compare the intrinsic ablation coefficients of stony material obtained empirically by Ceplecha \& ReVelle (2005) with theoretical values computed for melting and evaporation of stony meteoroids. Bronshten (1983, p. 123) gives the typical values for large stony meteoroids: $\xi=8 \mathrm{MJ} \mathrm{kg}^{-1}, \Lambda=0.1, \Gamma=0.46$. This gives the ablation coefficient $\sigma=\Lambda /(2 \xi \Gamma)=0.013 \mathrm{~s}^{2} \mathrm{~km}^{-2}$. However, the heat transfer coefficient, $\Lambda$, depends significantly on the meteoroid size, velocity, composition and height of flight. The numerical model of Golub' et al. (1996) gives $\Lambda=0.07-0.24$ for iron meteoroids of radii $0.1-1 \mathrm{~m}$ moving at the heights $30-40 \mathrm{~km}$ with the velocity $10-20 \mathrm{~km} \mathrm{~s}^{-1}$. The corresponding values for stony meteoroids should be lower (Bronshten et al. 1985). The model of Artemieva \& Shuvalov (2001) gives $\Lambda$ as low as 0.02 and 0.03 for stony body of $1 \mathrm{~m}$ radius and $20 \mathrm{~km} \mathrm{~s}^{-1}$ velocity at the heights 50 and $70 \mathrm{~km}$, respectively. If we consider the drag coefficient derived directly from the observation of the Lost City fireball and meteorite fall, $\Gamma=0.7 \pm 0.1$ (Ceplecha 1996), the possible values of the intrinsic ablation coefficient are between 0.002 and $0.025 \mathrm{~s}^{2} \mathrm{~km}^{-2}$. The observations of fireballs (Ceplecha \& ReVelle 2005; Borovička \& Kalenda 2003) favor the values closer to the lower edge. Note that small meteoroids in the transition flow regime are expected to have larger $\sigma$ $\left(\sigma \sim 0.05 \mathrm{~s}^{2} \mathrm{~km}^{-2}\right)$ because of larger $\Lambda(\Lambda \sim 0.8, \Gamma \sim 1)$. For a recent review of meteoroid ablation models see Popova (2005).

We have not yet included into consideration iron meteoroids, i.e. the nickel-iron metal material which constitutes iron meteorites. This is understandable because iron meteoroids form only few percent of fireballs and they do not appear clearly in a statistical analysis. Irons can be readily identified from their spectra but spectral records are available only for a minority of fireballs. ReVelle \& Ceplecha (1994) attempted to identify iron meteoroids from dynamic and photometric data. Because of their large thermal conductivity, irons in the mass range $10^{-3}$ to $10^{5} \mathrm{~kg}$ are expected to ablate by melting and to have larger ablation coefficients than chondritic material. The authors found 7 fireballs with smooth light curves, with no dynamically identifiable gross-fragmentation, and with large ablation coefficients (median value $0.05 \mathrm{~s}^{2} \mathrm{~km}^{-2}$ ), as probable irons. The 
large ablation coefficients are presumably intrinsic but it would be interesting to analyze those fireballs with the new FM method. According to the end height classification (PE criterion), irons fall into type II fireballs. The lower penetration ability of irons into the atmosphere in comparison with stony meteoroids can be well modeled using material properties of irons (Bronshten et al. 1985).

\section{Classification and ablation of small meteoroids}

\subsection{Classification}

Fainter meteors produced by meteoroids smaller than few centimeters have been classified according to their beginning heights (Ceplecha 1968, 1988). The classification was based on meteors photographed by sensitive Super-Schmidt cameras. These data cover meteoroid masses from about $10^{-5} \mathrm{~kg}$ to $10^{-2} \mathrm{~kg}$. The dependence of meteor beginning height on meteoroid mass was found to be not significant and a $K_{B}$ criterion was created, which relates the beginning height with initial velocity and trajectory slope only. Originally, four groups of small meteoroids have been recognized: A, B, C, D. These groups, however, do not correspond directly to the fireball groups. Group A corresponds to II, C to IIIA, and D to IIIB. The reason for different classifications are different proportions of asteroidal and cometary meteoroids in diferent mass ranges. Asteroidal bodies of group I are virtually absent below 1 gram of mass (Ceplecha 1988). On the other hand, the intermediate group B has no analog among large bodies.

Modern observations of faint meteors with image intensified TV cameras have shown that the meteor beginning height in facts depends on meteoroid mass. Koten et al. (2004) studied the beginning heights of meteors of five meteor showers. With the exception of Geminids, they found a definite dependence of the beginning height, $h_{\mathrm{b}}$, on the meteoroid mass. The dependence could be fitted with a function $h_{\mathrm{b}}=h_{0}+k \log m$, with $k$ ranging between 5 and $10 \mathrm{~km}$ for Orionids, Taurids, Perseids and Leonids in the mass range $10^{-7}$ to $10^{-4} \mathrm{~kg}$. Similar dependence with $k=6 \mathrm{~km}$ was found for 1998 Leonids in the mass range $10^{-8}$ to $10^{-5} \mathrm{~kg}$ by Campbell et al. (2000), though the authors considered the dependence as weak. Sarma \& Jones (1985) found $k=5$ and $k=9 \mathrm{~km}$, respectively, using two different camera systems on sporadic meteors of the similar mass range. Brown et al. (2000) found $k=9 \mathrm{~km}$ for single station 1999 Leonids.

The remarkable dependence of beginning height on mass does not mean that the meteor groups found by Ceplecha (1968) do not exist. Neither it means that the original data or the analysis were wrong. The Super-Schmidt cameras used blue sensitive Xray films (Millman 1959), while the image intensifiers have maximum sensitivity in the yellow-green part of the spectrum and their sensitivity extends far into infrared region (e.g. Borovicka et al. 1999). By chance, the blue region of the spectrum contains mostly emissions from the evaporated meteoric atoms, while the red and infrared radiation is produced mostly by heated air (Borovicka et al. 1999). This may be the basis for different meteor behavior with different detectors. We will discuss meteor begining heights in more detail in Sect. 3.4. In any case, the $\mathrm{K}_{\mathrm{B}}$ criterion cannot be used for TV meteors unless the considered mass range is narrow.

\subsection{Ablation coefficients}

Bellot Rubio et al. (2002) performed dynamic and photometric analysis of Super-Schmidt meteors. They found that data on $73 \%$ of the studied 370 meteors can be fitted with the single body equations, i.e. no fragmentation is needed to explain the data. However, they did not fit directly the length as a function of time but used the interpolated velocities and decelerations from the original catalog. These data were available at only few points 
along the trajectory for some meteors and photometric data were available at only four points for all meteors. The resulting median ablation coefficients of Bellot Rubio et al. (2002) are quite large: $0.07 \mathrm{~s}^{2} \mathrm{~km}^{-2}$ for $\mathrm{C}$ and $\mathrm{B}$ groups and $0.11 \mathrm{~s}^{2} \mathrm{~km}^{-2}$ for A group. Most of $\mathrm{C}$ group meteors $(85 \%)$ could be explained by single body theory, while for A group the percentage was only $44 \%$.

The large ablation coefficients of Super-Schmidt meteors are the result of neglecting fragmentation in my opinion. These are the apparent ablation coefficients. The only surprise is the larger median value for the A group than for the B and $\mathrm{C}$ groups. The distribution of ablation coefficients was, however, quite wide, from 0.01 to at least $0.20 \mathrm{~s}^{2} \mathrm{~km}^{-2}$ for all groups.

\subsection{Light curves}

TV observations of meteors provide good light curves, though no deceleration data. The light curves have been a subject of several detailed studies in the recent years (e.g. Murray et al. 1999, 2000; Koten et al. 2004). These studies concentrated on Leonids and other meteor showers mostly of cometary origin. The light curves of faint meteors are smooth with no flares. Most light curves have only one maximum. On the other hand, the shapes of the light curves vary very much from meteor to meteor, even for meteors of similar brightness belonging to the same shower. The maximum can lie almost anywhere on the light curve. On average, the light curves are nearly symmetrical with the maximum in the middle of the trajectory. These findings contradict the single body theory which produces uniform light curves with gradual increase and rapid decrease of brightness and the maximum at $70 \%$ of the trajectory (e.g. Beech \& Murray 2003). The observed light curves can be much better interpreted in terms of the dustball model formulated by Hawkes \& Jones (1975).

In the dustball model, a cometary meteoroid consists of grains of a high boiling-point material (e.g. stone or iron). The grains are held together by some binding material (glue) of a lower boiling point. The luminosity of the meteor is supposed to be produced only by ablating grains. The grains can start to ablate after they have been released from the dustball, which occurs when the glue surrounding the grain reached the boiling temperature. The grains released high in the atmosphere take some time before they start to ablate and radiate. The grains released deeper in the atmosphere start to radiate very quickly. Meteoroids smaller than certain critical mass release all grains before the grain ablation starts. The main prediction given by Hawkes \& Jones (1975) are the meteor heights. The beginning height should be the same for all meteors of given velocity and composition, while the end height and the height of maximum brightness should be constant for meteoroids smaller than the critical mass and should decrease with mass for larger meteoroids.

Beech \& Murray (2003) computed synthetic light curves of dustball meteors which release all grains before the onset of ablation. A power law distribution of the grain masses was assumed. More specifically, a $10^{-6} \mathrm{~kg}$ Leonid meteoroid was assumed to be composed of grains in the mass range $10^{-7}$ to $10^{-10} \mathrm{~kg}$. The synthetic light curve was simply the sum of light curves of individual grains. Light curves of various shapes were obtained by varying the mass distribution index, $\alpha$. For $\alpha$ close to 2 , early peaked light curves were obtained (maximum at 35\% of the trajectory). For smaller and larger $\alpha$ the light curve approached that of a single body. A few observed double peaked light curves could also be explained by adding a large mass grain to otherwise power law distribution (see also Murray et al. 1999).

A similar but more elaborated model was presented by Campbell-Brown \& Koschny (2004). Their model allows for gradual release of grains from the dustball and can be 
used for direct fitting of observed light curves. The mass distribution of grains is assumed to be either Gaussian or power law or a combination of both. The temperatures at which the grains were released in three Leonid meteors were found to lie between 1000-1150 K. In addition, Koschny et al. (2002) presented a model with Poisson distribution of grain sizes. The model can generate light curves with quite early maxima.

Another independently developed concept is the analytical model of quasi-continuous fragmentation (Babadzhanov 2002 and references therein). There are several processes possible which can cause quasi-continuous separation of small fragments from the meteoroid during the flight - the release of grains from a relatively large dustball is one of them. Babadzhanov (2002) fitted the light curves of relatively bright photographic meteors (meteoroid masses $>10^{-5} \mathrm{~kg}$ ) with this model. 111 out of 197 meteors could be fitted, the remaining light curves (44\%) did not conform with the QCF model. The light curves of quasi-continuously fragmenting meteoroids are not very different from single body light curves. Both observations and theory show that large meteoroids have light curves more similar to a single body. For example, the maxima of all Perseids larger than $10^{-5} \mathrm{~kg}$ lie in the second half of the light curve (Koten et al. 2004). This does not mean that the structure of larger meteoroids is different than smaller ones. Larger meteoroids are simply not disrupted completely before the grain ablation starts.

Statistically, there are differences among meteoroids of different streams. The average position of Leonid maximum brightness is in the middle of the light curve. Geminids of similar mass range reach their maxima at $58 \%$ of the trajectory (Koten et al. 2004). This can mean that Geminids, on average, are less fragile than Leonids. Nevertheless, as noted above, there is significant spread of light curves within one meteor shower.

\subsection{Beginning heights}

One prediction of the dustball theory, namely that the meteor beginning height is independent on meteoroid mass, could not be confirmed (see Sect. 3.1). Only for Geminids is the beginning height nearly constant (Koten et al. 2004). The increasing beginning heights of Leonids gradually merge into the extreme beginning heights of bright Leonid fireballs (Koten et al. 2006). The beginnings of Leonid fireballs were found to lie substantially higher when observed with TV systems than photographically observed beginnings (Fujiwara et al. 1998) and can reach $200 \mathrm{~km}$ (Spurný et al. 2000a). There is a continuous increase of Leonid beginning heights from $110 \mathrm{~km}$ for meteoroids of $10^{-7} \mathrm{~kg}$ to $200 \mathrm{~km}$ for meteoroids of $1 \mathrm{~kg}$. The high beginnings are not restricted to Leonids but occur also in other fireballs (Koten et al. 2001, 2006; Spurný et al. 2005), though up to now they have been observed only in fast fireballs of cometary origin.

The appearance of meteors above the height of $130 \mathrm{~km}$ differs from that at lower heights. Above $130 \mathrm{~km}$ the meteors are very diffuse, the luminous volume is several $\mathrm{km}$ wide and shows irregular structures (Spurný et al. 2000b; LeBlanc et al. 2000). Also the light curve shows irregularities (Spurný et al. 2000b; Koten et al. 2006). The high altitude radiation was originally considered as enigmatic. New studies, however, show that both the total luminosity and the size of the radiating volume can be explained (Ceplecha \& ReVelle 2005; Popova et al. 2005a; Vinković 2005). The physical mechanism responsible for the effect is sputtering of meteoroid surface by incoming atmospheric atoms and molecules, as first suggested by Brosch et al. (2001) and described in more detail by Rogers et al. (2005). This ablation mechanism works for fast meteors before the meteoroid surface reaches boiling temperature. Sputtering is negligible for velocities lower than $30 \mathrm{~km} \mathrm{~s}^{-1}$ (Popova et al. 2005a). At higher velocities, sputtering works for chondritic material but is more efficient for meteoroids containing more volatiles. The luminous volume is formed by cascade collisions of the sputtered atoms with surrounding atmospheric 
species (Vinković 2005). The main contributor to the radiation is oxygen triplet at $777 \mathrm{~nm}$ (Popova et al. 2005a; Spurný et al. 2005). The interaction of the sputtered atoms with the atmosphere can be perhaps more effectively traced by the produced ionization. Brosch et al. (2001) detected meteor echoes at heights $>200 \mathrm{~km}$ with a powerful phasedarray radar during Leonid maxima.

Koten et al. (2004) showed that the increase of beginning heights with meteoroid mass can be explained if the beginning height is given by the limiting sensitivity of the instrument and the ablation and radiation in fact starts earlier than the meteor is detected. Indeed, Campbell-Brown \& Koschny (2004) predicted a gradual brightening of the meteor after they abandoned the concept of boiling temperature in their model and used the Clausius-Clapeyron equation to calculate the mass loss. The increasing meteor beginning heights are therefore not in contradiction with the dustball model, only with its part which predicts that meteor radiation starts only after the grains reach their boiling temperature. The crucial question is whether the early ablation needs a significant volatile component to be present in meteoroids. In theory, both sputtering and the Clausius-Clapeyron equation allows high altitude ablation of pure stone. On the other hand, a volatile component (e.g. the glue in the dustball model) will enhance the ablation rate and the early meteor luminosity, even if the volatiles contribute to the radiation only indirectly by the collisions with the air. From the observational point of view it is significant that Geminids do not show beginning increase with meteoroid mass (Koten et al. 2004). One may argue that this due to their relatively low velocity $\left(35 \mathrm{~km} \mathrm{~s}^{-1}\right)$. However, even slower Taurids do show the increase, though it is less pronounced than for $71 \mathrm{~km} \mathrm{~s}^{-1}$ Leonids (Koten et al. 2004). It seems therefore likely that not only the high velocity sputtering but also gradual evaporation of a volatile component contributes to the luminosity of cometary meteors before the onset of regular grain ablation.

\subsection{Differential ablation}

The situation is complicated by the fact that even the ablation of regular chondritic material may not be uniform. Thermodynamic equilibrium calculation shows that fractionation occurs during melting and vaporization (McNeil et al. 2002; Schaefer \& Fegley 2005). More volatile atoms, in particular $\mathrm{Na}$, vaporize earlier than $\mathrm{Mg}, \mathrm{Fe}$, and $\mathrm{Si}$, while refractory $\mathrm{Ca}$ vaporizes later on and not fully. This fact was used to create a model of meteor differential ablation (McNeil et al. 1998), which was used to explain much higher abundance of $\mathrm{Na}$ than $\mathrm{Ca}$ in atmospheric metal layers. The model received some support from probing of fresh meteor trails by atmospheric lidars (von Zahn et al. 1999, 2002). The lidars rarely saw two or three elements simultaneously at the same position in the meteor trails. If they did, the observed elemental ratio was different from the expected chondritic value. The differences were larger for smaller meteoroids. The observations were done for $\mathrm{K}, \mathrm{Fe}$, and $\mathrm{Ca}$, which have very different volatility.

Differential ablation should be detectable by optical spectroscopy of faint meteors. Sensitive low resolution TV spectrographs detect bright lines of $\mathrm{Na}$ and $\mathrm{Mg}$ and fainter lines of $\mathrm{Fe}$ and $\mathrm{Ca}$ emitted by the material vaporized from the meteoroid (Borovicka et al. 1999, Abe et al. 2000). Potassium is not easily detectable. In Leonids, the Na line was often starts and ends earlier than the Mg line Borovicka et al. 1999). This is exactly what the model of differential ablation predicts. However, the effect of early release of Na varies from meteor to meteor and although it is present to some degree in the majority of Leonids, in some cases it is absent (Fig. 2). The situation is more complex if sporadic meteors and other showers are considered. In Taurids, for instance, Na closely follows $\mathrm{Mg}$ (Borovička 2001). On the other hand, an excellent example of early $\mathrm{Na}$ release was 




Figure 2. Monochromatic $\mathrm{Mg}, \mathrm{Na}$, and $\mathrm{O}$ light curves of two Leonid meteors of similar brightness $(\sim 0 \mathrm{mag})$. The oxygen line intensity was divided by 5 for better comparison. The heights are approximate.

observed in a photographic Draconid spectrum (Millman 1972). Draconids belong to the slowest meteor showers, so the effect is not a function of velocity.

The effect of early sodium release is not universal and must depend on meteoroid structure. It is reasonable to suppose that the quick evaporation of $\mathrm{Na}$ from the whole volume requires initial disruption of the millimeter-sized meteoroid - in accordance with the dustball model. Sodium can be part of the glue. Nevertheless, it can be also part of the constituent grains. For small grains, the complete fractionation and Na evaporation from the whole volume is probably possible. In any case, the early Na release is an indication of meteoroid disruption well before it was heated. The differences of the $\mathrm{Na}$ behavior in different meteors are probably due to different disruption height. Detailed correlation of spectral data with other meteor diagnostics - light curve shapes, beginning heights, end heights etc. - is desirable for better understanding of the behavior and structure of small meteoroids.

The differential ablation of calcium will be discussed in Sect. 7 .

\section{Bulk densities}

Meteoroid bulk density is a parameter of large interest. The different penetration ability of asteroidal and cometary meteoroids in the atmosphere may be primarily due to their different densities and it is desirable to know the absolute values of the densities. The derivation of meteoroid density from meteor data is, however, not straightforward and various authors used various approaches.

As noted in Sect. 2.2, the fireball dynamic analysis provides the product $K^{-1 / 3}$. If the light curve is taken into consideration and a correct luminous efficiency, $\tau$, is employed, the mass, $m$ can be separated from the shape-density coefficient, $K . K$ itself is a combination of the drag coefficient, $\Gamma$, shape coefficient, $A$, and bulk density, $\delta$. In their most recent model, Ceplecha \& ReVelle (2005) were reluctant to compute $\delta$ since, strictly speaking, $\Gamma$ and $A$ are unknown. In their model, $K$ showed large variations and it is difficult to say to which quantity the variations should be ascribed. In fact, the variations of $K$ could also be caused by the inappropriateness of the used $\tau$ for the given fireball. 
Table 2. Average bulk densities for different meteoroid groups (Ceplecha et al. 1993).

\begin{tabular}{lc}
\hline Group & Bulk density $\left[\mathrm{g} \mathrm{cm}^{-3}\right]$ \\
\hline I & 3.7 \\
II & 2.0 \\
IIIA & 0.75 \\
IIIB & 0.4 \\
\hline
\end{tabular}

Table 3. Average bulk densities for different meteors showers as determined by two authors.

\begin{tabular}{lcc}
\hline Shower & \multicolumn{2}{c}{ Bulk density $\left[\mathrm{g} \mathrm{cm}^{-3}\right]$} \\
& Babadzhanov $(2002)$ & Bellot Rubio et al. $(2002)$ \\
\hline Geminids & 2.9 & 1.9 \\
$\delta$ Aquarids & 2.4 & \\
Quadrantids & 1.9 & 0.8 \\
Taurids & 1.5 & 0.4 \\
Perseids & 1.3 & 0.6 \\
$\alpha$ Capricornids & & 0.45 \\
Leonids & 0.4 & \\
\hline
\end{tabular}

In the earlier gross-fragmentation model, Ceplecha et al. (1993) assumed $K$ to be constant and the light curve was not modeled in detail, only the initial mass was computed from the light curve. The resulting average bulk densities for meteoroids of different groups generally confirmed the earlier estimations (Ceplecha 1988) and are given in Table 2. Note that the IIIA and IIIB values are based on one fireball only for each group. They are nevertheless close to the earlier estimations $\left(0.75\right.$ and $0.27 \mathrm{~g} \mathrm{~cm}^{-3}$, respectively). The same gross-fragmentation model was also applied to several Geminid fireballs (Ceplecha \& McCrosky 1992) resulting in an average density of $3.3 \mathrm{~g} \mathrm{~cm}^{-3}$ (assuming $\Gamma A=1.1$ ). Geminid meteoroids therefore belong to group I, which was also demonstrated on the basis of their apparent ablation coefficients (Spurný 1993). One of the Leonid fireball observed by Spurný et al. (2000a) showed measurable deceleration. The bulk density was estimated to $0.7 \mathrm{~g} \mathrm{~cm}^{-3}$ (again for $\Gamma A=1.1$ ), close to the IIIA average. The apparent ablation coefficient was $0.16 \mathrm{~s}^{2} \mathrm{~km}^{-2}$. The modeling of the light curve and deceleration of the very bright IIIB Šumava fireball yielded the most probable bulk density as low as $0.1 \mathrm{~g} \mathrm{~cm}^{-3}$ (Borovička \& Spurný 1996).

The modeling of Super-Schmidt meteors by Bellot Rubio et al. (2002) described in Sect. 3.2 yielded also meteoroid bulk densities. They were computed from the resulting $K$ coefficient assuming $\Gamma=1$ and $A=1.21$ (spherical shape). The resulting densities for individual meteoroids show large scatter, from $0.1 \mathrm{~g} \mathrm{~cm}^{-3}$ to $4.5 \mathrm{~g} \mathrm{~cm}^{-3}$. The mean density was $2.4 \mathrm{~g} \mathrm{~cm}^{-3}$ for A-group meteoroids, $1.4 \mathrm{~g} \mathrm{~cm}^{-3}$ for group B, and $0.4 \mathrm{~g} \mathrm{~cm}^{-3}$ for group C. The authors also computed the mean bulk densities for different meteor showers (Table 3). As noted in Sect. 3.2, the work of Bellot Rubio et al. (2002) suffered from relative paucity of data points for individual meteors and from neglecting the fragmentation in the model. We consider their densities underestimated, in particular for group C.

Various attempts were published to determine meteoroid bulk densities using the model of quasi-continuous fragmentation (see Sect. 3.3). The results are sensitive on the tuning of various parameters of the model. As described in Bellot Rubio et al. (2002), earlier works used too low specific energy of fragmentation resulting in too high densities. Babadzhanov (2002) used a more realistic energy $\left(2 \times 10^{6} \mathrm{~J} \mathrm{~kg}^{-1}\right)$ and obtained more 
reasonable densities (Table 3 ). Still, he had to assume several parameters (shape, heat transfer coefficient, grain density) and he fitted only the light curves, not decelerations of meteors. Similarly, Konovalova (2003) roughly fitted light curves of several Taurid fireballs though deceleration data were available. She obtained average Taurid density of $2.5 \mathrm{~g} \mathrm{~cm}^{-3}$ and fragmentation energy of $5 \times 10^{5} \mathrm{~J} \mathrm{~kg}^{-1}$.

As it can be seen from Table 3, the densities of Bellot Rubio et al. (2002) are systematically lower than those of Babadzhanov (2002). Both authors, nevertheless, agree that Geminid stream contains the densest meteoroids of all streams. We consider the absolute Geminid value of Babadzhanov (2002) more realistic since it is close to that of Ceplecha \& McCrosky (1992).

In summary, the measured meteoroid bulk densities cover a wide range from about $0.1 \mathrm{~g} \mathrm{~cm}^{-3}$ to $3.7 \mathrm{~g} \mathrm{~cm}^{-3}$. Iron meteoroids with densities of $\sim 7 \mathrm{~g} \mathrm{~cm}^{-3}$ certainly also exist but they have not been directly measured. Although the derivation of meteoroid density from meteor data is difficult and involves number of assumptions, I consider the obtained density range as realistic. It correspond to the range of bulk densities covered together by ordinary chondrites, carbonaceous chondrites and interplanetary dust particles (IDP's) (Rietmeijer \& Nuth 2000). There are no evidences of significant differences in composition of meteoroids of different bulk densities. As in the case of IDP's, the density differences are most probably due to differences in porosities. The porous aggregate IDP's have a mean density of only $0.1 \mathrm{~g} \mathrm{~cm}^{-3}$ and porosity of $95 \%$ (Rietmeijer \& Nuth 2000). As shown by the Sumava meteoroid, similar values are possible for bodies up to several meters in diameter. These considerations correspond with the work of ReVelle (2001), who argued that the differences in penetration ability and apparent ablation parameters among meteoroids of different types can be naturally explained by their different porosities. He derived the following porosities and bulk densities: $0 \%\left(3.7 \mathrm{~g} \mathrm{~cm}^{-3}\right)$ for group I, $50 \%\left(1.85 \mathrm{~g} \mathrm{~cm}^{-3}\right)$ for group II, $75 \%\left(0.93 \mathrm{~g} \mathrm{~cm}^{-3}\right)$ for group IIIA, and $91 \%\left(0.34 \mathrm{~g} \mathrm{~cm}^{-3}\right)$ for group IIIB.

\section{Mechanical strength}

The front surface of a meteoroid passing through the atmosphere with a velocity $v$ is subject to a dynamic pressure $p=\rho v^{2}$, where $\rho$ is the density of the atmosphere. More precisely, the pressure is $p=\Gamma \rho v^{2}$ but the drag coefficient $\Gamma$ is not precisely known and is of the order of unity, so we ignore it. The pressure on the rear surface is nearly zero. If the front pressure, $p$, exceeds the mechanical strength of the meteoroid, meteoroid break-up occurs. Depending on the internal structure of the body, the break-up can lead to the formation of two macroscopic fragments or to the cloud of tiny dust particles of to something in between. As shown above, there are various methods to reveal different kinds of meteoroid fragmentation: meteor flares, sudden changes in the dynamics, direct imaging of the fragments. As soon as the fragmentation height is determined, it is easy to compute the dynamic pressure at the moment of the fragmentation, since meteor velocity as a function of height is easily measurable.

We have to note that the fragmentation understood in this section is different from the grain release after the disruption of a small dustball meteoroid or the quasi-continuous fragmentation described in Sect. 3. Those processes were driven by the heating of the whole meteoroid or its surface. In this section, the fragmentation is caused by mechanical forces and affects hitherto unaffected meteoroid interior.

\subsection{Cometary meteoroids}

It is not surprising that low dynamic pressures are sufficient to break up porous cometary bodies. The five big disruptions of the Šumava meteoroid occurred under the pressures 
between 0.025 and 0.14 MPa (Borovička \& Spurný 1996). The fragmentation sequence could be described by a simple concept of destruction depth. Under this concept, the dynamic pressure acting on the surface of the meteoroid causes at some moment the destruction of the meteoroid to the depth $d$, which is proportional to the pressure $p$. The destroyed part is pulverized while the rest of the body remains more or less intact, suggesting low cohesivity of the material. This process repeats quasi-periodically until $d$ exceeds meteoroid radius and the body is destroyed completely, leaving only few small fragments to continue. The fragments are finally destroyed in the next step. This pattern may be general for IIIB meteoroids with the exception that smaller bodies do not have enough mass for such complex behavior as Šumava and only one flare may be present.

Taurid fireballs were observed to break-up under 0.05-0.18 MPa (Konovalova 2003). The onset of terminal flares of Leonid fireballs typically occurs between the heights 95$85 \mathrm{~km}$ (Spurný et al. 2000a; Borovička \& Jenniskens 2000). This corresponds to $p=$ 0.007-0.04 MPa. However, the effective lower limit of the strength of Leonid meteoroids may be close to zero. This statement is based on the observation of meteoroid clusters within the Leonid shower which were shown to be products of meteoroid fragmentation in the interplanetary space few days before the encounter with the Earth (Watanabe et al. 2003).

On the other hand, there are evidences that Leonid meteoroids contain also much stronger ingredients. In some cases, small part of the original mass does not participate in the 'terminal' flare and continues the flight deeper in the atmosphere. Examples of Leonid fireballs containing such a strong fragment are the LN98023 fireball of Spurný et al. (2000b) and the fireball analyzed by Borovička \& Jenniskens (2000). The former fragment penetrated to $73 \mathrm{~km}$ of height and experienced the pressure of $0.2 \mathrm{MPa}$, the latter penetrated down to $56 \mathrm{~km}$ and survived the pressure close to $2 \mathrm{MPa}$, which is quite a lot even in comparison with group I meteoroids. The surviving fragment comprised only a tiny part of the original mass $\left(\sim 10^{-6}\right)$ and its spectrum did not revealed any anomaly in the chemical composition. The size was of the order of $1 \mathrm{~mm}$. Swindle \& Campins (2004) speculated whether this is an evidence for presence of chondrules in cometary matter but considered it unlikely. Rather, this can be regarded as a piece of evidence for the opinion expressed by various authors (see e.g. Lodders \& Osborne 1999) that cometary nuclei contain also compact material analogous to (or identical to) CI and CM carbonaceous chondrites.

There are also other evidences for the existence of compact cometary material of different kind. Among the fireballs photographed by the European Fireball Network, the Karlštejn fireball (Spurný \& Borovička 1999a,b) is unique. The cometary origin is evident from the retrograde orbit (inclination $=139^{\circ}$ ), though the semimajor axis was unusually small $(a=3.5 \mathrm{AU})$. The fireball, however, penetrated much deeper than other cometary fireballs of similar velocity and mass and was classified as type I fireball. There is no flare on the light curve and the meteoroid survived dynamic pressure of $0.7 \mathrm{MPa}$, which is the lower limit of its mechanical strength. The meteoroid density was likely at least $2 \mathrm{~g} \mathrm{~cm}^{-3}$, the mass was of the order of hundreds of grams and the size reached several centimeters. The fireball spectrum revealed that the meteoroid was completely free of sodium, unlike normal cometary meteoroids. Similar Na-free meteoroids have been later found among small millimeter sized meteoroids on cometary orbits (Borovička et al. 2005). These meteoroids are possibly remnants of cometary irradiation crusts that formed during the comet residence in the Oort cloud. Meteor showers do not contain this material because their parent comets have lost their primordial irradiation crust long time ago. Centimeter-sized crust fragments are much rarer than the smaller ones but the Karlštejn meteoroid is a good example of their existence. 


\subsection{Asteroidal meteoroids}

The fragmentation of stronger bodies of groups I and II can be better studied by geometric and dynamic methods because the break-ups are not always accompanied by sizeable flares. Ceplecha et al. (1993) applied the dynamic method to 51 fireballs with sufficiently precise photographic records. The method was able to find fragmentation events where more than $\sim 50 \%$ of mass was lost. Nearly $60 \%$ of fireballs of groups I and II showed at least one fragmentation. (The model of Ceplecha \& ReVelle (2005), which takes into account all details on the light curve, often detects a dozen of small fragmetations for one fireball.) The dynamic pressures at fragmentation indicated some grouping around the values of $0.08,0.25,0.53,0.8$, and $1.1 \mathrm{MPa}$, which allowed Ceplecha et al. (1993) to define five strength categories, 'a' to 'e'. Three meteoroids survived 1.5 MPa without fragmentation and one meteoroid survived $5 \mathrm{MPa}$.

The work of Ceplecha et al. (1993) concerned ordinary fireballs corresponding to meteoroid sizes up to few decimeters. It is of interest to look at the largest bodies for which data are available, with the sizes of the order of one meter. Large bodies can fragment extensively - dozens of individual fragments can be seen on still photographs and videos of the Peekskill (Brown et al. 1994) and Morávka (Borovička \& Kalenda 2003) meteorite falls. The fragmentation can occur in several stages. The significant deceleration of the large meteoroids Benešov (Borovička et al. 1998) and Morávka (Borovička \& Kalenda 2003) at the heights of $\sim 45 \mathrm{~km}$ is the evidence for the fact that they have been already disrupted into a dozen of fragments of similar mass $(100-300 \mathrm{~kg})$ at that height. The disruption height could not be determined from the data, so only the upper limit for the dynamic pressure can be set: $p<0.5 \mathrm{MPa}$. This limit, nevertheless, is in full accordance with the values for smaller meteoroids (Ceplecha et al. 1993). The primary fragments of Benešov and Morávka continued a cascade fragmentation at lower heights. The dynamic pressures at that break-ups were up to $5 \mathrm{MPa}$ for Morávka and up to $9 \mathrm{MPa}$ for Benešov. Not all large bodies, nevertheless, are subject to the initial high altitude disruption. The 1.3 meter EN 171101 meteoroid (Spurný \& Porubčan 2002) survived intact until the pressure of $4 \mathrm{MPa}$ and the daughter fragments later experienced $14 \mathrm{MPa}$ without fragmentation, before reaching the record low terminal height of $13 \mathrm{~km}$.

Popova et al. (2005b) recently compiled the fragmentation data of the nine instrumentally observed meteorite falls and compared them with the published strengths of various meteorites. All nine meteoroids experienced fragmentation during the flight. The strongest bodies were Neuschwanstein (10 MPa) and Př́bram (14 MPa, uncertain value). The strengths of meteorites measured in the laboratory are significantly larger - the average tensile strength is $30 \mathrm{MPa}$ (range 2-60 MPa) and the compression strength, which is more relevant for atmospheric fragmentation, is $200 \mathrm{MPa}$ on average (20-450 MPa). This confirms that the recovered meteorites represent the strongest structural parts of the incoming meteoroids. The meteoroids, as asteroidal fragments, certainly experienced many collisions in their lifetime and contain cracks and other structural weakness. Most of the meteoroids therefore break easily apart under the dynamic pressures of 0.1-1 MPa. Only the strongest ones can resist up to the pressures of about $5 \mathrm{MPa}$. The meteoroid strength seems to be not a function of meteoroid mass, rather it depends on individual history of each body.

Of course, the more massive is the body, the more complex can be the atmospheric fragmentation. The most detailed fragmentation study was performed for the Morávka meteoroid (Borovička \& Kalenda 2003). The parameters of 15 fragmentation events observed at the heights of $32-24 \mathrm{~km}$ were determined from geometric and dynamic data. The complete disruption (mass loss larger than 90\%) was not observed. On the other 
Table 4. Meteoroid mechanical strengths inferred from fireball fragmentation data

\begin{tabular}{llr}
\hline Fireball(s) & Corresponding material/ parent body & Mechanical strength range [MPa] \\
\hline Sumava & weak cometary material, IIIB & $0.025-0.14$ \\
Taurids & comet 2P/Encke & $0.05-0.18$ \\
Leonids & comet 55P/Tempel-Tuttle - bulk material & $0-0.04$ \\
Leonids & primordial cometary crust (?) & $\geqslant 2$ \\
Karlštejn & type I fireballs & ordinary chondrites \\
Tagish Lake & carbonaceous chondrite, D-type asteroid & $0.08-14$ \\
\hline
\end{tabular}

hand, the sum of mass of continuing fragments was always markedly lower than the mass of the original body, indicating that part of mass was always lost in form of dust. One unexplained fact is that successive fragmentations occurred under lower dynamic pressures than the previous events. In addition,

The fragments of the Morávka meteoroid gained lateral velocities up to $300 \mathrm{~m} \mathrm{~s}^{-1}$ (Borovička \& Kalenda 2003) - an order of magnitude more than the aerodynamic theory (Artemieva \& Shuvalov 2001) predicts. The same fact was observed for fragmentation of cometary Taurid meteoroids at much larger heights $(65 \mathrm{~km})$ by Konovalova (2003). The Taurid lateral velocity reached $130 \mathrm{~m} \mathrm{~s}^{-1}$. As suggested by Konovalova (2003), the large lateral velocities could be explained by an explosive nature of the fragmentations, triggered by the exposition of explosive magnesium dust in the atmosphere. The rotational explanation of the fragmentation and fragment dispersion was also proposed, in particular for the Peekskill meteoroid (Beech \& Brown 2000), but I consider this less probable because of high rotation rate involved (see Sect. 6).

Among the observed meteorite fall, there is also the unique carbonaceous chondrite Tagish Lake. The bulk density of the meteorite, $1.7 \mathrm{~g} \mathrm{~cm}^{-3}$, is the lowest of all known meteorites (Zolensky et al. 2002). The material survived the atmospheric entry only owing to the large initial mass of the meteoroid, estimated to 56 tones and corresponding to diameter of 4 meters (Brown et al. 2002). The first significant fragmentation occurred under the dynamic pressure $0.25 \mathrm{MPa}$, the main break-up started at $0.7 \mathrm{MPa}$. The latter value probably corresponds to the compressive strength of the material (Brown et al. 2002). In other words, the fragmentation - unlike ordinary chondrites - cannot be ascribed to the presence of fractures. The meteoroid was in fact disrupted into thousands of pieces in this event. The accompanying flare and dust cloud created in the atmosphere are evidences for enormous dust release. Note that the Tagish Lake meteorite is spectrally similar to D-type asteroids (Hiroi et al. 2001).

The data presented in this section are summarized in Table 4.

\section{Rotation}

The classical equations of meteoroid motion through the atmosphere (e.g. Ceplecha et al. 1998) assume that meteoroid cross section decreases monotonically following the decrease of mass. In case of a non-spherical rotating meteoroid, there will be periodic variation of the head cross-section and this will induce periodic variation of both the mass loss rate and the atmospheric drag. In consequence, periodic terms in meteor light curve and decelerations could be observed. In addition, rapid rotation of a meteoroid (even spherical) will delay the onset of intensive evaporation because of slower heating of the surface and can, in theory, lead to meteor beginning heights by $10 \mathrm{~km}$ lower in 
comparison with non-rotating case (Adolfsson \& Gustafson 1994). This mechanism does not apply for very small meteoroids which are heated isothermally.

Periodic variation of fireball brightness are really observed from time to time in the form of rapid flickering. The observational data have been summarized by Beech \& Brown (2000). The flickering occurs in about $4 \%$ of bright fireballs. Typically, the flickering is seen at only a fraction of the trajectory, sometimes near the fireball beginning, sometimes near the end. There is no obvious correlation of height of flickering with meteoroid type or size. The reported flickering frequencies vary from as low as few $\mathrm{Hz}$ to as high as $500 \mathrm{~Hz}$. In some fireballs the frequency is constant but often it grows rapidly with time. The amplitudes are fairly constant and less than one magnitude. Beech \& Brown (2000) and Beech (2002) interpreted the flickering as a demonstration of meteoroid rotation. On the contrary, Babadzhanov \& Konovalova (2004) studied high frequency (> $100 \mathrm{~Hz}$ ) flickering of three Geminids and discarded the rotation hypothesis on the basis of the observed beginning heights and the fact that flickering started suddenly in the middle of the trajectory. They proposed an autofluctuation mechanism of ablation as the explanation of high frequency flickering. The question of the nature of fireball flickering remains open. I consider a fluctuating ablation mechanism as more likely explanation, at least for fast flickering of large meteoroids. Beech (2001) fitted the light curve of the Innisfree fireball (meteorite fall) with a low frequency $(2.5 \mathrm{~Hz})$ periodic term but the original photometric data are sparse and do not indicate periodicity, so this case is not convincing.

Possibly the most reliable value of meteoroid rotation is available for the thoroughly investigated Lost City fireball and meteorite fall (Ceplecha 1996; Ceplecha \& ReVelle 2005). The elongated meteoroid of the size $36 \times 17 \mathrm{~cm}$ rotated with the period of $3.3 \pm$ 0.3 seconds. This result was obtained by Adolfsson by studying the details of fireball dynamics, namely the residuals in the length as a function of time after applying the fragmentation model. Though the details have not been published, the method seems to be robust.

\section{Chemical composition}

The derivation of elemental abundances in meteoroids can be in principle done from meteor spectroscopy. Meteor spectra contain emission lines produced by the atoms evaporated from the meteoroid. The derivation of abundances poses some obvious problems. First, estimation of ionization and excitation conditions in meteor plasma is needed for converting line intensities into elemental abundances. Second, the number of observable elements is restricted by the used wavelength region and the quality of the spectrum. Third, because of the effects of differential ablation and incomplete evaporation (Sect. 3.5), the composition of the vapor at a given trajectory point may not reflect the bulk composition of the meteoroid.

The general picture of the excitation conditions in meteor plasma was obtained by Borovička $(1993,1994)$. Meteor radiation can be fitted by two spectral components, the main component of medium temperature (typically 4000-5000 K), and a high temperature component $(\sim 10,000 \mathrm{~K})$. Both components are assumed to be in thermal equilibrium, at least for larger meteoroids at lower heights $(<100 \mathrm{~km})$. This assumption explains the line intensities reasonably well and was also confirmed theoretically by direct Monte Carlo simulation of a $1 \mathrm{~cm}$ Leonid at a height of $95 \mathrm{~km}$ (Boyd 2000).

To determine the composition of the radiating vapors, it is desirable to fit the line intensities with thermal equilibrium model and determine the excitation temperature and column densities of observed species. In the next step, it is necessary to estimate the electron density and ionization degree of different elements. The procedure 
is best applied to high resolution photographic spectra of bright fireballs (Borovička 1993, 2005). Modern CCD detectors can provide comparable resolution but lower wavelength coverage (Jenniskens \& Mandell 2004, Jenniskens 2005). Kasuga et al. (2005a,b) were courageous enough to apply the procedure to low resolution TV spectra. Note that Kasuga et al. (2004) did not apply the ionization correction and they results cannot be considered.

\subsection{Large meteoroids}

The spectra of bright fireballs do not show large diversity in chemical composition of meteoroids. Iron meteoroids have been detected (Halliday 1960; Ceplecha 1966) but they are rare $(\approx 1 \%$ of bright meteors). A brief survey of 53 spectra from the Ondřejov archives revealed, besides the Ceplecha's (1966) iron, only one probable diogenite (Borovička 1994b). It is worth to mention that the heliocentric orbit put the diogenite almost exactly in the 3:1 resonance with Jupiter, consistent with the delivery from the Vesta asteroid family (Binzel \& Xu 1993). Since then, the Ondřejov observation yielded the unique Karlštejn meteoroid deficient in sodium (Spurný \& Borovička 1999b, see also Sect. 5.1), one possible eucrite (unpublished), and a fireball defficient in $\mathrm{Mg}$ and $\mathrm{Ca}$ (Borovička 2005b). A more extensive survey of fainter photographic meteors (Harvey 1973) yielded fewer than $10 \%$ of spectra suggesting anomalous (non-chondritic) chemical composition.

For more detailed consideration, quantitative analysis is needed. Borovička (1993) analyzed an excellent fireball spectrum at 43 points between the heights $57-35 \mathrm{~km}$. The composition of the radiating gas varied significantly along the trajectory. The refractory elements $\mathrm{Al}, \mathrm{Ti}$, and $\mathrm{Ca}$ showed the most pronounced variations. Their abundances increased at lower heights and in flares but never reached the chondritic values (when compared with Fe or $\mathrm{Mg}$ ). The effect was even more pronounced in the deeply penetrating Benešov fireball (Borovička \& Spurný 1996), where the relative Ca abundance increased by more than two orders of magnitude between the upper $(78 \mathrm{~km})$ and lower $(24 \mathrm{~km})$ part of the trajectory. The chondritic value was reached in the lower part. It is evident that refractory elements are evaporated incompletely in slow fireballs, unless the fireball reaches dense layers of the atmosphere. For high speed fireballs on Halley-type orbits, various degree of completeness of evaporation of Ca was found (Fig. 3). The incomplete evaporation of refractories is in agreement with thermodynamical calculations (McNeil et al. 2002; Schaefer \& Fegley 2005) and can explain low abundances (or non-detection) of Ca in meteor trails observed by lidars (von Zahn et al. 1999, 2002).

In Fig. 3 the abundances obtained by different authors are compiled. If values at different points along the trajectory were published, the part with most complete evaporation of refractories was taken. All included spectra belong to the 'normal' ones which suggest nearly chondritic composition at the first sight (one spectrum of Trigo-Rodriguez et al. (2003) with low Mg abundance was omitted). The quantitative results for cometary meteors on Halley type orbits (mostly Perseids and Leonids) show an interesting pattern. $\mathrm{Fe}, \mathrm{Cr}$, and $\mathrm{Mn}$ are depleted and $\mathrm{Si}$ and $\mathrm{Na}$ enhanced relative to $\mathrm{Mg}$ when compared with CI abundances. The same trend was measured by mass spectroscopy of the dust of comet Halley (Jessberger et al. 1988). The trend, except for the Na enhancement, is less pronounced for cometary meteors on ecliptical orbits. Unfortunately, the scatter of data is large. Trigo-Rodriguez et al. (2003) concluded (on basis of Mg-Fe-Si ratio) that cometary meteoroids have CI composition rather than that of Halley dust, while Borovička (2005a) claimed the opposite for Leonids and Perseids. Nevertheless, low Fe/Mg ratio was seen in Leonids also by Abe et al. (2005) and Kasuga et al. (2005a), and for smaller meteoroids by Borovička et al. (2005). 


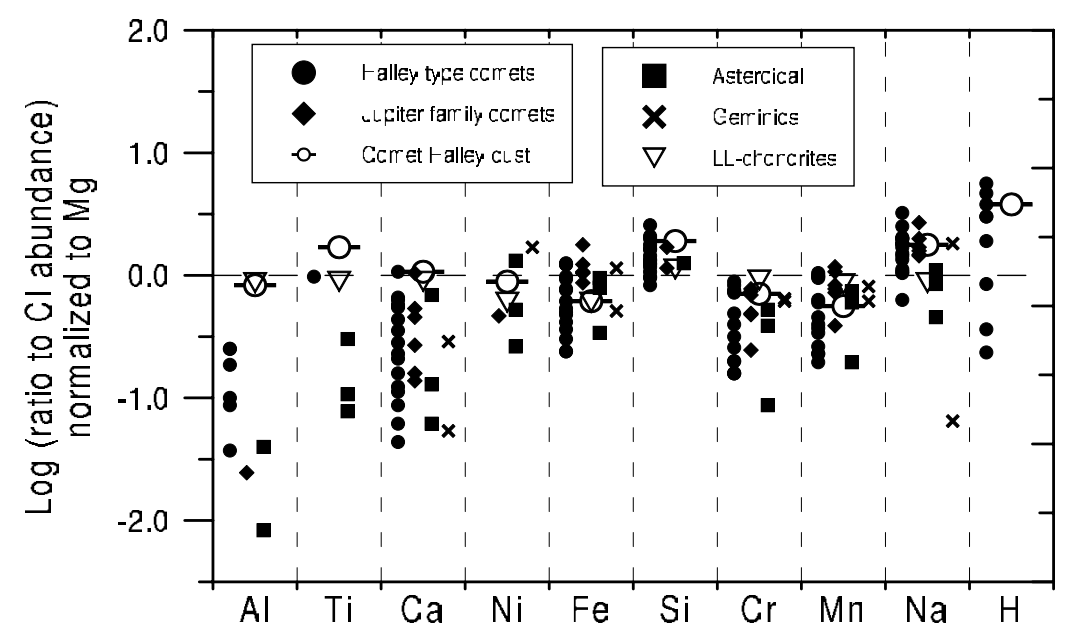

Figure 3. Elemental abundances derived from fireball spectra expressed as deviations from CI abundances, normalized to Mg. The fireballs are divided according to their origin. The volatility of the atoms increases from left to right. The in situ measured abundances of the dust of comet 1P/Halley (Jessberger et al. 1988) and of LL ordinary chondrites (Wasson \& Kallemeyn 1988) are plotted for comparison. CI abundances and volatilities taken from Lodders (2003). Fireball data taken from Borovička (1993), Borovička \& Spurný (1996), Borovička \& Betlem (1997), Trigo-Rodriguez et al. (2003), Kasuga et al. (2005a,b), and Borovička (2005a,b).

The difficulty of the interpretation is stressed by the fact that asteroidal fireballs also do not show chondritic composition. The abundances for LL chondrites are also given in Fig. 3 to show the range of chondritic values. The Benešov fireball (Borovička \& Spurný 1996) seems to be very Mg-rich. The other two asteroidal bodies show nearly chondritic $\mathrm{Fe} / \mathrm{Mg}$ and $\mathrm{Na} / \mathrm{Mg}$ ratios. $\mathrm{Cr}$, however, is underabundant, though to less extent than in Halley-type cometary meteoroids. The fact that $\mathrm{Cr}$ shows systematic variations along the fireball trajectory (Borovička 1993) suggests that an unidentified process can cause depletion of atomic $\mathrm{Cr}$ in the radiating gas.

The possibility that the composition of Halley dust is representative also for large cometary meteoroids remains open but the decision lies at the boundary of the resolution of present spectroscopic methods.

\subsection{Small meteoroids}

A survey of spectra of meteor produced by small (millimeter sized) meteoroids was published by Borovička et al. (2005). Small meteoroids were found much more diverse than large ones. Based on the intensities of the $\mathrm{Mg}, \mathrm{Na}$, and Fe lines, only a minority of sporadic meteoroids have chondritic composition. The large diversity of small meteoroids in comparison with larger ones may reflect real inhomogeneity of comets on millimeter scale. Alternatively, environmental effects such as solar wind can alter small meteoroids.

Three populations of Na-free meteoroids were found. The first population consists of iron meteoroids originating in the main asteroid belt, probably related to iron meteorites. This population, surprisingly, forms majority of asteroidal meteoroids in this size range. Millimeter sized ordinary chondrites are rare, which was also found from non-spectral study by Ceplecha (1988). The second population of Na-free meteoroids consist of meteoroids on orbits with small perihelia $(q \leqslant 0.2 \mathrm{AU})$. The loss of $\mathrm{Na}$ was caused by thermal processes in the vicinity of the Sun and was accompanied by general compaction of the body. The meteoroids of the $\delta$ Aquarid stream belong to this population. Geminids, which also have small perihelia, show variable content of $\mathrm{Na}$ (see also Fig. 3). This is 
likely caused by varying ages of the meteoroids. The third population of Na-free meteoroids resides on Halley type orbits. These bodies are probably remnants of cometary irradiation crust and are related to the Karlštejn meteoroid (Spurný \& Borovička 1999b; Sect. 5.1).

Fe-poor meteoroids with normal content of $\mathrm{Na}$ were also identified. All of them have low material strength and come from comets. Even cometary meteoroids classified as normal, in particular Leonids, showed partial depletion of Fe. Sporadic meteoroids on Halley type orbits are, nevertheless, much more diverse than shower meteoroids. The content of Fe and $\mathrm{Na}$ varies widely in cometary meteoroids. The reasons are not quite clear but may include various ages, differences among parent comets, and different processes involved in their release from comets.

\subsection{Organic matter, water}

Cometary meteoroids are a potential source of organic matter (Jenniskens et al. 2004). Effort has been made in the recent years to search for organic elements (CHON) in the spectra of meteors, in particular Leonids. Oxygen and nitrogen are readily seen in meteor spectra, their primary source is, however, the atmosphere. Hydrogen can be seen in the high temperature component of the spectra of fast fireballs. The derived $\mathrm{H} / \mathrm{Mg}$ abundance varies widely, from less than in CI chondrites to somewhat more than in Halley dust (Fig. 3). In addition to data given in Fig. 3, Borovička \& Jenniskens (2000) estimated $\mathrm{H} / \mathrm{Fe}=10-20$ and Jenniskens \& Mandell (2004) gave $\mathrm{H} / \mathrm{Si}=4$, which both certainly falls into the range given in Fig. 3. The large scatter of $\mathrm{H}$ abundances may be partly real. At least some meteoroids contain significant part of hydrogen. The hydrogen may come either from water embedded in partly hydrated minerals Rietmeijer 2005) or from organic material. Water ice is not expected to survive one perihelion passage in 0.1-m sized Leonid or Perseid meteoroids (Beech \& Nikolova 2001). Pellinen-Wannberg et al. (2004) claimed to detect water in an Leonid but their evidence is very weak and indirect. Carbon has been positively identified in a UV Leonid spectrum (Carbary et al. 2003) but the abundance has, unfortunately, not been computed.

In the visual region, carbon is more likely to be detected in molecular form. The search for the main band of $\mathrm{CN}$ at $388 \mathrm{~nm}$ in Leonid spectra was unsuccessful, yielding a limit $\mathrm{CN} / \mathrm{Fe} \leqslant 0.03$ (Jenniskens et al. 2004). The authors proposed an explanation that carbon is ablated in form of more complex molecules. Long time ago, Ceplecha (1971) detected $\mathrm{CN}$ and possibly also $\mathrm{C}_{2}$ in the terminal flare of of a bright sporadic cometary fireball. His resolution in the $388 \mathrm{~nm}$ region was, however, lower than that of Jenniskens et al. (2004). The ultraviolet band of $\mathrm{OH}$ at $308 \mathrm{~nm}$ was tentatively detected by Jenniskens et al. (2002) and Abe et al. (2005). Russell et al. (2000) detected CO, $\mathrm{CO}_{2}, \mathrm{H}_{2} \mathrm{O}$ and probably $\mathrm{CH}_{4}$ in the mid-infrared spectrum of a meteor train several minutes after the fireball passage. These species are, however, unlikely to come directly from the meteoroid. At the present the content of organic matter in meteoroids is not firmly established.

\section{Summary}

There are various populations of meteoroids coming to the Earth. Asteroidal fragments capable to drop stony meteorites are well represented among large meteoroids. Their bulk mechanical strength, however, is typically two orders of magnitude smaller than that of recovered meteorites. Stony meteoroids of all sizes are full of cracks and do fragment easily during their atmospheric entry. Atmospheric fragmentation must be taken into account when considering the impacts of small asteroids on the Earth (Bland \& Artemieva (2003); Melosh \& Collins 2005). Stony meteoroids smaller than about $1 \mathrm{~cm}$ are rare in 
the interplanetary space. On the other hand, iron meteoroids, which form only $\sim 1 \%$ of large bodies, are surprisingly well represented among millimeter sized meteoroids.

Cometary meteoroids are present at all sizes. They differ from the asteroidal bodies mainly by their large porosity and low bulk density. Two types of cometary material have been recognized, one with density about $0.7 \mathrm{~g} \mathrm{~cm}^{-3}$ and the other with $0.1-0.4 \mathrm{~g} \mathrm{~cm}^{-3}$. Both types are formed by loosely bound grains of various sizes and are likely analogical to aggregate IDP's and porous aggregate IDP's, respectively. The grains may be held together by a volatile glue (matrix) but the existence of the glue has not been confirmed with certainty. In any case, the grains can be released under moderate heating. The accumulated pressure acting on very large cometary meteoroids can blow away the material to certain depth but the meteoroid can withstand complete destruction, suggesting that cometary material is not fully cohesive. Fragile cometary meteoroids can, nevertheless, hide small more resistant chunks, possibly similar to carbonaceous chondrites.

The ablation ability and chemical composition of cometary grains does not largely differ from chondritic material. There is a hint that Halley type comets are richer in Na, $\mathrm{Si}$, and $\mathrm{Mg}$ and poorer in $\mathrm{Fe}, \mathrm{Cr}$, and $\mathrm{Mn}$ in comparison with chondrites. The meteoroids coming from active comets are chemically relatively homogenous. Larger diversity is found among sporadic meteoroids of cometary origin and sizes of several millimeters. Part of these are fragments of cometary irradiation crust. They are depleted in volatiles $(\mathrm{Na})$ and are significantly stronger than normal cometary material. The loss of volatiles and general compaction also occurs in the vicinity of Sun. Small meteoroids with perihelia within $0.2 \mathrm{AU}$ are chemically and physically altered. This process can be at least partly responsible for the high density of Geminid meteoroids.

\section{Acknowledgements}

This work was supported by grant no. 205/05/0543 from GAČR. My research is conducted under the project ASCR project AV0Z10030501.

\section{References}

Abe, S., Yano, H., Ebizuka, N., \& Watanabe, J. 2000, Earth, Moon and Planets 82-83, 369

Abe, S., Ebizuka, N., Yano, H., Watanabe, J., \& Borovička, J. 2005, Astrophys. J. 618, L141

Adolfsson, L.G. \& Gustafson, B.Å.S. 1994, Planet. Space Sci. 42, 593

Artemieva, N.A. \& Shuvalov, V.A. 2001, J. Geophys. Res. 106 (E2), 3297

Arvidson, R.E. \& Squyres, S.W. 2005, Amer. Geophys. Union, Spring Meeting 2005, abstract \#P31A-02

Babadzhanov, P.B. 2002, Astron. Astrophys. 384, 317

Babadzhanov, P.B. \& Konovalova, N.A. 2004, Astron. Astrophys. 428, 241

Beech, M. 2001, Mon. Not. R. Astron. Soc. 326, 937

Beech, M. 2002, Mon. Not. R. Astron. Soc. 336, 559

Beech, M. \& Brown, P. 2000, Planet. Space Sci. 48, 925

Beech, M. \& Murray, I.S. 2003, Mon. Not. R. Astron. Soc. 345, 696

Beech, M. \& Nikolova, S. 2001, Planet. Space Sci. 49, 23

Bellot Rubio, L.R., Martínez González, M.J., Ruiz Herrera, L. et al. 2002, Astron. Astrophys. 389,680

Binzel, R.P. \& Xu, S. 1993, Science 260, 186

Bland, P.A. \& Artemieva, N.A. 2003, Nature 424, 288

Borovička, J. 1993, Astron. Astrophys. 279, 627

Borovička, J. 1994a, Planet. Space Sci. 42, 145

Borovička, J. 1994b, in: Y. Kozai et al. (eds.) Seventy-Five Years of Hirayama Asteroid Families, Astron. Soc. Pacific Conf. Ser. 63, p. 186 
Borovička, J. 2001, in: B. Warmbein (ed.), Proc. Meteoroids 2001 Conf., ESA-SP 495, p. 203

Borovička, J. 2005a, Earth, Moon and Planets (in press)

Borovička, J. 2005b, IAU Symp. 229 Abstract

Borovička, J. \& Betlem, H. 1997, Planet. Space Sci. 45, 563

Borovička, J. \& Jenniskens,P. 2000, Earth, Moon and Planets 82-83, 399

Borovička, J. \& Kalenda, P. 2003, Meteorit. Planet. Sci. 38, 1023

Borovička, J. \& Spurný, P. 1996, Icarus 121, 484

Borovička, J., Popova, O.P., Nemtchinov, I.V., Spurný, P., \& Ceplecha, Z. 1998, Astron. Astrophys. 334, 713

Borovicka, J., Stork, R. \& Bocek, J. 1999, Meteorit. Planet. Sci. 34, 987

Borovička, J., Koten, P., Spurný, P., Boček, J., \& Štork, R. 2005, Icarus 174, 15

Boyd, I.D. 2000, Earth, Moon and Planets 82, 93

Bronshten, V.A. 1983, Physics of Meteoric Phenomena (Dordrecht: Reidel)

Bronshten, V.A., Rabunskij, D.D., \& Tertitskij, M.I. 1985, Astron. Vestnik 19, 224

Brosch, N., Schijvarg, L.S., Podolak, M., \& Rosenkrantz, M.R. 2001, in: B. Warmbein (ed.), Proc. Meteoroids 2001 Conf., ESA-SP 495, p. 165

Brown, P., Ceplecha, Z., Hawkes, R.L., Wetherill, G., Beech, M., \& Mossman, K. 1994, Nature 367,624

Brown, P., Campbell, M.D., Ellis, K.J., Hawkes, R.L., Jones, J., Gural, P., Babcock, D., Barnaum, C., Bartlett, R.K., \& Bedard, M. and 31 more authors 2000, Earth, Moon and Planets 82, 167

Brown, P.G., ReVelle, D.O., Tagliaferri, E., \& Hildebrand, A.R. 2002, Meteorit. Planet. Sci. 37, 661

Campbell, M.D., Brown, P.G., LeBlanc, A.G. et al. 2000, Meteorit. Planet. Sci. 35, 1259

Campbell-Brown, M.D. \& Koschny, D. 2004, Astron. Astrophys. 418, 751

Carbary, J.F., Morrison, D., Romick, G.J., \& Yee, J.-H. 2003, Icarus 161, 223

Ceplecha, Z. 1966, Bull. Astron. Inst. Czech. 17, 195

Ceplecha, Z. 1968, Smithson. Astrophys. Obs. Spec. Rep. 279

Ceplecha, Z. 1971, Bull. Astron. Inst. Czech. 22, 219

Ceplecha, Z. 1988, Bull. Astron. Inst. Czech. 39, 221

Ceplecha, Z. 1996, Astron. Astrophys. 311, 329

Ceplecha, Z. \& McCrosky, R.E. 1976, J. Geophys. Res. 81, 6257

Ceplecha, Z. \& McCrosky, R.E. 1992, in: A. W. Harris, E. Bowell (eds.), Asteroids, Comets, Meteors 1991 (Huston: Lunar Planet. Inst. Houston), p. 109

Ceplecha, Z. \& ReVelle, D.O. 2005, Meteorit. Planet. Sci. 40, 35

Ceplecha, Z., Spurný, P., Borovička, J., \& Keclíková, J. 1993, Astron. Astrophys. 279, 615

Ceplecha, Z., Borovička, J., Elford, W.G., ReVelle, D.O., Hawkes, R.L., Porubčan, V., \& Šimek, M. 1998, Space Sci. Rev. 84, 327

Cook, A.F., \& Duxbury, T.C. 1981, J. Geophys. Res. 86, 8815

Cudnik, B.M., Dunham, D.W., Palmer, D.M., et al. 2003, Earth, Moon and Planets 93, 145

Fujiwara, Y., Ueda, M., Shiba, Y. et al. 1998, Geophys. Res. Lett. 25, 285

Golub', A.P., Kosarev, I.B., Nemchinov, I.V., \& Shuvalov, V.V. 1996, Astron. Vestnik 30, 213

Halliday, I. 1960, Astrophys. J. 132, 482

Harvey, G.A. 1973, in: C.L. Hemenway et al. (eds.), Evolutionary and Physical Properties of Meteoroids, NASA-SP 319, p. 131

Hawkes, R.L. \& Jones, J. 1975, Mon. Not. R. Astron. Soc. 173, 339

Hiroi, T., Zolensky, M.E., \& Pieters, C.M. 2001, Science 293, 2234

Jacchia, L.G., Kopal, Z., \& Millman, P.M. 1950, Astrophys. J. 111, 104

Jenniskens, P. 2005, Adv. Space Res. (submitted)

Jenniskens, P. \& Mandell, A.M. 2004, Astrobiology 4, 123

Jenniskens, P., Tedesco, E., Murthy, J., Laux, C.O., \& Price, S. 2002, Meteorit. Planet. Sci. 37, 1071

Jenniskens, P., Schaller, E.L., Laux, C.O., Wilson, M.A., Schmidt, G., \& Rairden, R.L. 2004, Astrobiology 4, 67

Jessberger, E.K., Christoforidis, A., \& Kissel, J. 1988, Nature 332, 691 
Kasuga, T., Watanabe, J., Ebizuka, N., Sugaya, T., \& Sato, Y. 2004, Astron. Astrophys. 424, L35

Kasuga, T., Yamamoto, T., Watanabe, J., Ebizuka, N., Kawakita, H., \& Yano, H. 2005a, Astron. Astrophys. 435, 341

Kasuga, T., Watanabe, J., \& Ebizuka, N. 2005b Astron. Astrophys. 438, L17

Konovalova, N.A. 2003, Astron. Astrophys. 404, 1145

Koschny, D., Reissaus, P., Knöfel, A., Trautner, R., \& Zender, J. 2002, in: B. Warmbein (ed.), Asteroid, Comets, Meteors (ACM2002), ESA-SP 500, p. 157

Koten, P., Spurný, P., Borovička, J., \& Štork, R. 2001, in: B. Warmbein (ed.), Proc. Meteoroids 2001 Conf., ESA-SP 495, p. 119

Koten, P., Borovička, J., Spurný, P., Betlem, H., \& Evans, S. 2004, Astron. Astrophys. 428, 683

Koten, P., Spurný, P., Borovička, J. et al. 2006, Meteorit. Planet. Sci. (submitted)

LeBlanc, A.G., Murray, I.S., Hawkes, R.L., Worden, P., Campbell, M.D., Brown, P., Jenniskens, P., Correll, R.R., Montague, T., \& Bavcock, D.D. 2000, Mon. Not. R. Astron. Soc. 313, L9

Lodders, K. 2003, Astrophys. J. 591, 1220

Lodders, K. \& Osborne, R. 1999, Space Sci. Rev. 90, 289

McNeil, W.J., Lai, S.T., \& Murad, E. 1998, J. Geophys. Res. 103 (D9), 10899

McNeil, W.J., Murad, E., \& Plane, J.M.C. 2002, in: E. Murad, I.P. Williams (eds.), Meteors in the Earth's Atmosphere (Cambridge: Cambridge University Press), p. 265

Melosh, H.J. \& Collins, G.S. 2005, Nature 434, 157

Millman, P.M. 1959, J. R. Astron. Soc. Canada 53, 15

Millman, P.M. 1972, J. R. Astron. Soc. Canada 66, 201

Murray, I.S., Hawkes, R.L., \& Jenniskens, P. 1999, Meteorit. Planet. Sci. 34, 949

Murray, I.S., Beech, M., Taylor, M.J., Jenniskens, P., \& Hawkes, R.L. 2000, Earth, Moon and Planets 82, 351

Pellinen-Wannberg, A., Murad, E., Gustavsson, B., Brädström, U., Enell, C.-F., Roth, C., Williamns, I.P., \& Steen, A. 2004, Geophys. Res. Lett. 31, L03812

Popova, O.P. 2005, Earth, Moon and Planets (in press)

Popova, O.P., Strelkov, A.S., \& Sidneva, S.N. 2005a, Adv. Space Res. (submitted)

Popova, O.P. et al. 2005b, IAU Symp. 229 Abstract

ReVelle, D.O. 2001, in: B. Warmbein (ed.), Proc. Meteoroids 2001 Conf., ESA-SP 495, p. 513

ReVelle, D.O. \& Ceplecha, Z. 1994, Astron. Astrophys. 292, 330

Rietmeijer, F.J.M 2005, Earth, Moon and Planets (in press)

Rietmeijer, F.J.M. \& Nuth III, J.A. 2000, Earth, Moon and Planets 82, 325

Rogers, L.A., Hill, K.A., \& Hawkes, R.L. 2005, Planet. Space Sci. 53, 1341

Russell, R.W., Rossano, G.S., Chatelain, M.A., Lynch, D.K., Tessensohn, T.K., Abendroth, E., Kim, D., \& Jenniskens, P. 2000, Earth, Moon and Planets 82, 439

Sarma, T. \& Jones, J. 1985, Bull. Astron. Inst. Czech. 36, 9

Schaefer, L. \& Fegley Jr., B. 2005, Earth, Moon and Planets (in press)

Selsis, F., Lemmon, M.T., Vaubaillon, J., \& Bell III, J.F. 2005, Nature 435, 581

Spurný, P. 1993, in: J. Štohl \& I.P. Williams (eds.), Meteoroids and Their Parent Bodies (Bratislava: Astron. Inst. Slovak Acad. Sci.), p. 193

Spurný, P. 1995, Earth, Moon and Planets 68, 529

Spurný, P. \& Borovička, J. 1999a, in: W.J. Baggaley \& V. Porubčan (eds.), Meteoroids 1998 (Bratislava: Astron. Inst. Slovak Acad. Sci.), p. 143

Spurný, P. \& Borovička, J. 1999b, in: J. Svoreň et al. (eds.), Evolution and Source Regions of Asteroids and Comets (Tatranská Lomnica: Astron. Inst. Slovak Acad. Sci.), p. 163

Spurný, P. \& Porubčan, V. 2002, in: B. Warmbein (ed.), Asteroid, Comets, Meteors (ACM2002), ESA-SP 500, p. 269

Spurný, P., Betlem, H., van't Leven, J., \& Jenniskenns, P. 2000a, Meteorit. Planet. Sci. 35, 243

Spurný, P., Betlem, H., Jobse, K., Koten, P., \& van’t Leven, J. 2000b, Meteorit. Planet. Sci. 35, 1109

Spurný, P., Borovička, J., \& Koten, P. 2005, Earth, Moon and Planets (in press)

Swindle, T.D. \& Campins, H. 2004, Meteorit. Planet. Sci. 39, 1733 
Trigo-Rodriguez, J.M., Llorca, J., Borovička, J., \& Fabregat, J. 2003, Meteorit. Planet. Sci. 38, 1283

Vinković, D. 2005, Adv. Space Res. (in press)

von Zahn, U., Gerding, M., Höffner, J., McNeil, W.J., \& Murad, E. 1999, Meteorit. Planet. Sci. 34,1017

von Zahn, U., Höffner, J., \& McNeil, W.J. 2002, in: E. Murad, I.P. Williams (eds.), Meteors in the Earth's Atmosphere (Cambridge: Cambridge University Press), p. 149

Wasson, J.T. \& Kallemeyn, G.W. 1988, Phil. Trans. R. Soc. Lond. A 325, 535

Watanabe, J., Tabe, I., Hasegawa, H., Hashimoto, T., Fuse, T., Yoshikawa, M., Abe, S., \& Suzuki, B. 2003, Publ. Astron. Soc. Japan 55, L23

Zolensky, M.E., Nakamura, K., \& Gounelle, M. 2002, Meteorit. Planet. Sci. 37, 737 